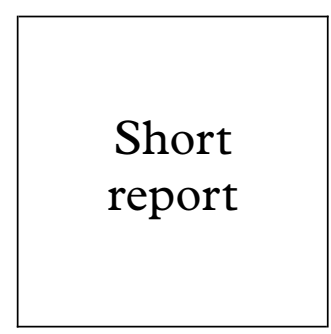

\title{
Sensitivity of the ligase chain reaction assay for detecting Chlamydia trachomatis in vaginal swabs from women who are infected at other sites
}

\author{
B J Thomas, T Pierpoint, D Taylor-Robinson, A M Renton
}

Objective: To assess the sensitivity of the ligase chain reaction (LCR) assay for Chlamydia trachomatis in vaginal swabs from women who were positive in cervical samples and/or urines.

Subjects: 413 women attending the genitourinary medicine clinic, St Mary's Hospital, Paddington. Methods: The LCR assay was used to test vaginal swabs from 46 women who were $C$ trachomatis positive at one or both of the other sites by direct fluorescent antibody (DFA) staining, by an enzyme immunoassay (EIA), or by the LCR assay.

Results: The LCR assay of vaginal swabs had the following sensitivity values using confirmed positive results: 93\% (41/44) compared with DFA staining of cervical deposits, 93\% (41/44) compared with the LCR assay of cervical samples, 93\% (28/30) compared with an EIA for cervical samples, 91\% (39/43) compared with DFA staining of urine deposits, and 93\% (39/42) compared with the LCR assay of urine. Four women had vaginal swab samples negative by the LCR assay; one was positive only in the urine and two had cervical samples containing a small number of chlamydial elementary bodies.

Conclusion: Testing vaginal swabs by the LCR assay is a sensitive method of detecting chlamydial infection; the results suggest that this procedure could be used as an alternative to examining urines in a screening programme for chlamydial infection in the community.

(Sex Transm Inf 1998;74:140-141)

Keywords: chlamydial infection; vaginal swabs; ligase chain reaction

\section{Introduction}

The performance of the ligase chain reaction (LCR) (Abbott) assay for Chlamydia trachomatis in urine samples ${ }^{12}$ makes it the likely method of choice for population screening programmes. However, the concentration of antigen in urine from some women can be low, ${ }^{34}$ and the sensitivity of the LCR assay for urine from pregnant women has been called into question. ${ }^{5}$ We have investigated the value of vaginal swab specimens as an alternative. The sensitivity of the LCR assay for vaginal samples has been determined in women whose cervical swab or urine samples were $C$ trachomatis positive by the LCR assay, by a direct fluorescent antibody (DFA) test, or by an enzyme immunoassay (EIA).

\section{Materials and methods}

SUBJECTS

We recruited 413 women attending the Jefferiss Wing genitourinary medicine clinic, St Mary's Hospital, Paddington who had not taken antibiotics in the previous month. Informed consent was obtained from all subjects.

\section{SAMPLES}

A vaginal swab sample was taken without the use of a speculum and placed in LCR transport medium. A speculum was then inserted and the cervix swabbed four times. As routine clinic procedure, the first swab sample was used for Gram staining and then plated on to GC agar and the second was placed in transport medium for the MicroTrak Chlamydia EIA (Behring). Two further swabs were taken for the study; the first was placed in transport medium for the $C$ trachomatis LCR assay and the second in phosphate buffered saline (PBS) which was centrifuged for DFA staining (MicroTrak; Behring). A first pass urine specimen was then obtained.

Two $C$ trachomatis positive women who had no vaginal swab samples taken were excluded from the study. Three women provided no urine sample and another had only a vaginal

Centre for Research, Charing Cross and Westminster Medical

School, London A M Renton

Correspondence to: Dr B Thomas, Department of Genitourinary Medicine The Winston Churchill Wing, Imperial College School of Medicine at St Mary's, Paddington, London W2 $1 \mathrm{NY}$.

Table 1 Comparison of the results of the LCR assay of vaginal swabs (VS) samples with those of DFA staining and the $L C R$ assay of cervical samples $(n=45)$ and urines $(n=43)$ and an EIA of cervical samples $(n=44)$ from 46 $C$ trachomatis positive women

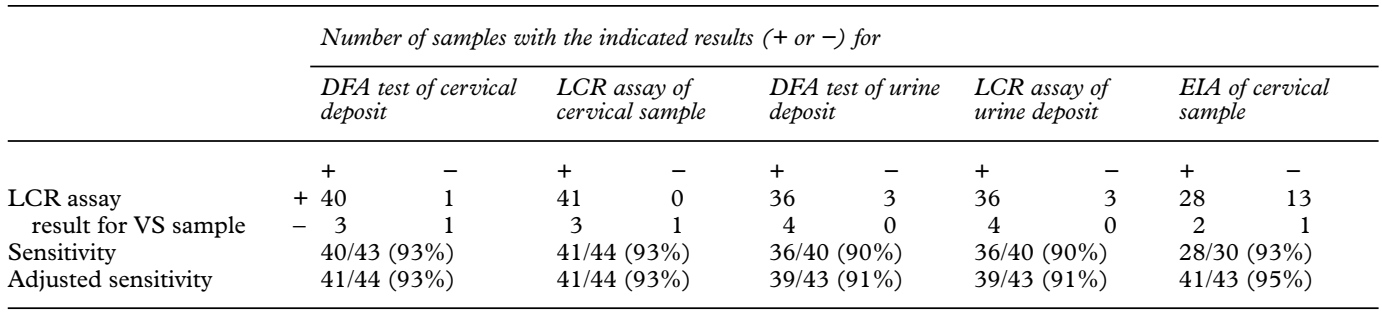


Table 2 Discrepant results of testing urine and cervical samples from patients whose vaginal swab (VS) samples by the LCR assay were positive $(n=6)$ or negative $(n=4)$

\begin{tabular}{|c|c|c|c|c|c|c|}
\hline \multirow[b]{2}{*}{ Patient no } & \multicolumn{6}{|c|}{ Assay result/site } \\
\hline & $E I A / c x$ & $D F A / c x$ & $L C R / c x$ & DFA/urine & LCR urine & $L C R / V S$ \\
\hline 418 & + & + & + & $+^{\star}$ & - & + \\
\hline 306 & - & + & + & $+^{\star}$ & - & + \\
\hline 502 & + & $+^{\star}$ & + & $t^{\star}$ & - & + \\
\hline 21 & $\mathrm{Eq}+\dagger$ & $+^{\star}$ & + & - & + & + \\
\hline 393 & + & + & + & - & + & + \\
\hline 256 & $\mathrm{Eq}+\dagger$ & - & + & - & + & + \\
\hline 432 & - & - & - & $+^{\star}$ & + & - \\
\hline 92 & + & $+^{\star}$ & + & $t^{\star}$ & + & - \\
\hline 37 & $\mathrm{Eq}++$ & $+\star$ & + & + & + & - \\
\hline 121 & + & + & + & $+^{\star}$ & + & - \\
\hline
\end{tabular}

*Sample contained $<10$ EBs by DFA staining. †Equivocal positive result.

swab sample for the LCR assay and a cervical sample for the EIA taken.

\section{MICROBIOLOGICAL TESTS}

DFA test

Cervical swab samples in PBS and urines were centrifuged and the deposits stained. ${ }^{3}$ The number of elementary bodies per sample was recorded as $<10,10-100,100-1000,>1000$, or $>10000$. A sample containing one or more elementary bodies was considered positive.

EIA test

This was performed on cervical samples by the diagnostic microbiology laboratory, St Mary's Hospital, Paddington.

\section{LCR assay}

This was performed according to the manufacturer's instructions. Samples were frozen at $-20^{\circ} \mathrm{C}$ for up to 14 days before being tested.

EIA results were recorded only for women who were $C$ trachomatis positive by another test; vaginal swab samples were tested by the LCR assay only for women who were $C$ trachomatis positive at another site.

\section{Results}

Forty eight $(11.6 \%)$ of the 413 women had at least one sample (cervix and/or urine) that was $C$ trachomatis positive by at least one test (EIA/ DFA/LCR); vaginal swab samples were available from 46 of these positive women and 42 $(91 \%)$ were positive by the LCR assay. The sensitivity values of the LCR assay for vaginal swab samples compared with the results of testing samples from other sites by the LCR assay or by the other methods are shown in table 1 .

In six patients, the LCR assay result for the vaginal swab sample was positive when another site or sites were negative by one of the other assays (table 2). Three urines were negative by the LCR assay, two urines were negative by DFA staining, and one patient had both urine and cervical samples negative by DFA staining. In all these patients, however, it was considered that the positivity of the LCR assay result for the vaginal swab sample was confirmed by positive results at other sites. The adjusted sensitivity values for the LCR assay of vaginal swab samples using the confirmed positive results are shown in table 1 .

Four women with negative vaginal swab samples were positive at other sites, one by DFA and LCR tests in the urine only, and two at all other sites, but with $<10$ elementary bod- ies in their cervical deposits by DFA staining. However, a further eight women whose cervical samples contained this small number of elementary bodies had positive LCR assay results for their vaginal swab samples.

Testing cervical samples by the EIA had a sensitivity of only $64 \%$ (28/44) compared with LCR testing of vaginal swab samples and $66 \%$ $(30 / 45 ; 29 / 44$, respectively) compared with the LCR assay and DFA testing of cervical samples. Two patients had unequivocally positive EIA results, confirmed by other tests, when their vaginal swab samples were negative by the LCR assay.

\section{Discussion}

Testing vaginal samples by the LCR assay is a sensitive method of diagnosing chlamydial infection. The results compare well with testing cervical samples and urines by the LCR assay and DFA staining. The results indicate that self taken vaginal swabs, tested in this way, might be used in community screening for chlamydial infection.

A previous study has shown that vulval swabs might also be suitable ${ }^{6}$ and work in Sweden has demonstrated $93 \%$ and $78 \%$ sensitivity, respectively, for mailed vaginal samples taken by pipette and mid stream urines tested by the LCR assay. The low sensitivity for urines was attributed to lack of refrigeration during mailing ${ }^{7}$ and current work in our laboratory confirms that the sensitivity of the LCR assay is reduced when urines are kept at room temperature, particularly those containing a small number of chlamydial elementary bodies. Vaginal swabs could provide an alternative to this problem of maintaining the cold chain for urines, and to the reportedly unsatisfactory performance of the LCR assay for urines from pregnant women. ${ }^{5}$ It is unlikely that vaginal swab samples from pregnant women are similarly affected, but this remains to be determined.

It is likely that a screening programme for $C$ trachomatis would reduce morbidity and decrease the incidence and prevalence of this organism in the population. The current results indicate this should not be undertaken by use of an EIA. However, the LCR assay, and other future molecular diagnostic tests, and the use of suitable and easily obtainable samples should make it achievable.

1 Schachter J, Moncada J, Whidden R, et al. Noninvasive tests for diagnosis of Chlamydia trachomatis infection: application of ligase chain reaction to first catch urine specimens of tion of ligase chain reaction to first catch

2 Chernesky MA, Jang D, Lee HH, et al. Diagnosis of Chlamydia trachomatis infections in men and women by Chlamydia trachomatis infections in men and women by testing first-void urine by

Microbiol 1994;32:2682-5.
Hay PE, Thomas BJ, Horner PJ, et al. Chlamydia trachomatis in women: the more you look, the more you find. Genitourin Med 1994;70:97-100.

4 Sellors JW, Mahony JB, Jang L, et al. Comparison of cervical, urethral and urine specimens for the detection of Chlamydia trachomatis in women. F Infect Dis 1991;164:205-8.

5 Jensen I, Thorsen P, Moller B. Sensitivity of ligase chain reaction assay of urine from pregnant women for Chlamydia trachomatis. Lancet 1997;349:329-30

6 Stary A. DNA amplification, antigen detection tests, and culture: which sample for which technique? In: Stary A, ed. Proceedings of the Third Meeting of the European Society for

7 Ostergaard L, Moller JK, Andersen B, et al. Diagnosis of Ostergaard L, Moller JK, Andersen B, et al. Diagnosis of
urogenital Chlamydia trachomatis infection in women based on mailed samples obtained at home: multipractice comparative study. BMF 1996;313:1186-9. 